\title{
Cross-Cultural Adaptation And Pilot Testing Of The Cancer Care Coordination Questionnaire For Patients (CCCQ-P) In Chinese And Arabic Languages
}

This article was published in the following Dove Press journal:

Patient Preference and Adherence

Jane $M$ Young $\mathbb{D}^{1-3}$

Rebecca L Venchiarutti ${ }^{1,2}$

Ivana Durcinoska ${ }^{1,2}$

Daniel Steffens (iD) ${ }^{1,2}$

'Surgical Outcomes Research Centre (SOuRCe), Royal Prince Alfred Hospital, Camperdown, NSW, Australia; ${ }^{2}$ Faculty of Medicine and Health, Sydney School of Public Health, The University of Sydney, Camperdown, NSW, Australia; ${ }^{3}$ RPA Institute of Academic Surgery, Sydney Local Health District, Camperdown, NSW, Australia
Correspondence: Jane M Young Surgical Outcomes Research Centre, Royal Prince Alfred Hospital, P.O. Box MI57, Missenden Road, Camperdown, NSW 2050, Australia

Email Jane.Young@sydney.edu.au
Background: The Cancer Care Coordination Questionnaire for Patients (CCCQ-P) has been designed to measure patients' experience of this crucial aspect of their cancer care. Migrants are at particular risk of receiving poorly coordinated cancer care due to challenges in communication as well as unfamiliarity with the health system and roles of health professionals. The aim of this study was to cross-culturally adapt and pilot test the CCCQ-P in Chinese and Arabic languages.

Methods: This study followed an established five-stage process for cross-cultural adaptation of self-report measures. The CCCQ-P was forward and back-translated into Arabic, Simplified Chinese, and Traditional Chinese languages by two independent translators. An expert committee review panel appraised the translations, resulting in a pre-final version in the target languages. Face validity, content validity, and consistency of the translated CCCQ$\mathrm{P}$ were then assessed in a sample of bilingual former cancer patients and health professionals. In addition, structured interviews were conducted to explore the meaning of each question and responses to participants.

Results: Thirteen health professionals (7 Chinese, 6 Arabic) and 19 former cancer patients (11 Chinese, 8 Arabic) participated in the face validation. Across both language groups, participants agreed that the cross-culturally adapted and translated versions had clear instructions and response options that were appropriate and understandable. All items were considered important and significant to the tool and so no item was removed. Complex medical words caused some differences in preferred terminology in Arabic and Chinese; however, participants agreed that the meaning of the questions and response options was not lost.

Conclusion: The Arabic, Simplified Chinese, and Traditional Chinese cross-culturally adapted and piloted versions of the CCCQ-P are useful tools to measure patients' experience of cancer care coordination. Further validation and psychometric testing of the instrument are warranted.

Keywords: care coordination, migrants, cancer

\section{Background}

Effective coordination of care is fundamental to the provision of high-quality patient-centred health care. ${ }^{1,2}$ For patients with cancer, optimal management requires input from multiple specialties to facilitate patient decision-making, streamlining access to and receipt of care, and improving psychosocial outcomes, 
while still prioritising appropriate curative or palliative treatment. Multidisciplinary management of cancer care requires access to a variety of services, in hospital and community settings, and across private and public sectors. Navigating a complex and often fragmented health care system can be challenging, particularly when faced with a cancer diagnosis, the prospect of treatment, and ongoing care that can extend for many years after treatment ends. ${ }^{3}$ Successful coordination of care is therefore essential to optimize patients' outcomes and ensure patients do not "fall through the gaps" of the health system. Patients are ideally placed to assess the adequacy of the coordination of the cancer care they have received, and in recent years, there has been a shift toward using patient-reported outcome measures (PROMs) as standard tools to assess patient health, outcomes, and experiences within the health system. $^{4}$

Australian population statistics reflect an increasing culturally diverse nation. The 2016 Australian Census ${ }^{5}$ indicated that $28 \%$ of the Australian population was born overseas, $21 \%$ had at least one parent born overseas, and $21 \%$ spoke a language other than English at home, figures that increased from the previous census in $2011 .^{6}$ In New South Wales (NSW), the most populous state in Australia, these figures were even higher, with $34 \%$ of the residents born overseas. ${ }^{5}$ These rapidly changing demographics present a challenge for health care providers to ensure equitable cancer care for migrant patients in an unfamiliar health care system.

Migrants face unique challenges to accessing resources and services that are culturally relevant and languageappropriate to navigate the health care system. ${ }^{7}$ Migrants are likely to be unfamiliar with a new and foreign health care system, often relying heavily on friends and family to overcome cultural and language barriers, and may feel confused about the cancer care system and the role of health professionals within cancer teams. ${ }^{7}$ In general, patients with a poor understanding of the health care system also have poorly coordinated cancer care, as demonstrated in a population-based study of colorectal cancer patients in NSW, Australia, ${ }^{1}$ and the added barrier of an unfamiliar health system may increase the risk for migrant patients. This may also explain the higher levels of clinical depression and anxiety, unmet information, supportive care or physical needs, and poorer quality of life of immigrant cancer patients in Australia compared to Anglo-Australians. ${ }^{8,9}$ However, most non-English speakers are excluded from measurement of coordination of cancer care due to the lack of availability of languagespecific instruments.

Studies have reported conflicting associations between race or ethnicity and care coordination. ${ }^{1,10}$ Ayanian et al ${ }^{11}$ surveyed Chinese-speaking patients with lung and colorectal cancer, reporting that, compared with other ethnic groups, these patients reported much worse scores of coordination and responsiveness of care, as well as physician communication and nursing care. Furthermore, Chinese-speaking patients were much less likely to give an overall rating of their care as "excellent" across both cancers. ${ }^{11}$ This indicates a palpable divide in the experiences of migrants and non-migrant cancer patients.

Over the past 10 years, our research group has developed a patient self-report questionnaire to measure their experience of care coordination - the Cancer Care Coordination Questionnaire for Patients (CCCQ-P). The 20 -item instrument was developed and validated in English in a series of iterative studies using rigorous methods and provides an overall score for care coordination experience as well as scores for two subscales ("communication" and "navigation"). ${ }^{12}$ In light of the lack of language-specific tools for non-English speakers to measure cancer care coordination, we set out to cross-culturally adapt and translate the English CCCQ-P into two of the most common languages other than English spoken in Australia, Chinese and Arabic. ${ }^{5}$ Therefore, the aim of this study was to translate, cross-culturally adapt, and pilot test the CCCQ-P into Arabic, Traditional Chinese, and Simplified Chinese languages.

\section{Methods}

\section{The CCCQ-P Instrument}

The CCCQ-P measures cancer care coordination across two domains: communication (13 items) and navigation (7 items). ${ }^{12}$ Participants' responses are measured on a fivepoint Likert scale assessing their level of agreement with each item (strongly agree, agree, neutral, disagree, or strongly disagree). Total scores range from 20 to 100 , with a higher score indicating better coordination of cancer care. In addition, there are two global assessment questions in which participants rate the coordination of their care, and quality of their care on a Likert scale from one (very poor) to ten (excellent). The CCCQ-P has previously been validated in 686 patients who had been recently treated for a newly diagnosed cancer. The entire instrument demonstrated high internal consistency (Cronbach's 
alpha 0.88), as well as individual subscales (Cronbach's alpha 0.87 and 0.73 for communication and navigation subscales, respectively). There were moderate correlations between each of the subscales and the two global measures, ranging from 0.41 to $0.57 .^{12}$ To improve the relevance of the instrument for migrant patients, culturally and linguistically diverse (CALD)-specific items, developed from focus groups and interviews with Chinese-, Macedonian-, and Arabic-speaking participants were added to the questionnaire ${ }^{7}$ alongside the original CCCQ-P instrument. The CALD-specific items included questions relating to access to language-specific resources and interpreters, understanding of the Australian health system, and agreement between Australian doctors and doctors in the patients' home country.

\section{Translation And Cross-Cultural Adaptation Of Questionnaire}

The translation and cross-cultural adaptation process followed the first five stages of the guidelines developed by Beaton et al. ${ }^{13}$ The stages involved in this process were as follows:

\section{Stage I - Translation}

Pairs of translators that were native speakers of either Simplified and Traditional Chinese or Arabic produced two independent forward translations of the original CCCQ-P instrument. One translator in each pair was required to have familiarity with medical terminology and an understanding of health care, while the other had no clinical background or experience.

\section{Stage 2 - Synthesis}

A single forward translation into the target language was produced during a reconciliation teleconference involving the core research team (content experts), a language-specific investigator (third native speaker), and the two forward translators.

\section{Stage 3 - Back Translation}

The forward translation was then back-translated into English by two independent translators blinded to the original English version of the CCCQ-P instrument.

\section{Stage 4 - Expert Committee Review}

An expert committee review, involving the core research team, language-specific investigator, and two translators, discussed the forward and back translations to achieve consensus on a single translated version of the CCCQ-P.
The review team discussed the semantic, idiomatic, experimental, and conceptual equivalence of the translations, resulting in a pre-final translated version of the CCCQ-P questionnaire in the target language.

\section{Stage 5 - Pilot Testing Of Pre-Final Questionnaire}

In this stage, the face validity of the pre-final questionnaires was examined by patients and health professionals to explore the meaning of the translated items and responses.

\section{Eligibility Criteria And Participant Recruitment}

Participants were eligible if they: were aged $\geq 18$ years, had been diagnosed with any malignant solid tumour within the last five years, were at least six months posttreatment completion, or they were a health professional (doctor, nurse, allied health staff), and were bilingual in English and either Chinese or Arabic. Patients who had a newly diagnosed or recurrent cancer at the time of the questionnaire administration, were in follow-up for haematologic cancers, were receiving end of life care, were cognitively impaired to be unable to provide written informed consent, or were not able to communicate in English were excluded. Patients were identified by their surgeon or oncologist at follow-up appointments, where they were given an explanation of the study. Alternatively, patients were identified by consultant surgeons or oncologists from patient records and sent an invitation to participate in the study and study materials to return in a provided pre-paid return addressed envelope. Health professionals were identified by study investigators on hospital wards or through professional networks. Bilingual health professionals were invited to provide feedback on the face validity of the pre-final questionnaire as these clinicians have experience in conversing with migrant patients in the Australian health care setting and would be able to provide unique insight into the phrasing of difficult medical terminology.

\section{Ethical Approval And Written Informed Consent}

Ethical approval to conduct the pilot testing for the pre-final questionnaire was obtained from the Sydney Local Health District (SLHD) Human Research Ethics Committee (HREC) (protocol number X17-0230). Clinical governance was approved by the Research Governance Office at the 
Royal Prince Alfred Hospital. Written informed consent was obtained from all participants in this study.

\section{Data Collection}

Participants were asked to read the pre-final version of the CCCQ-P in either Arabic or Chinese (Traditional or Simplified) and were provided with the original English version for reference. Provision of the English version in addition to the translated versions allowed participants to compare the translations to the English version and ensure that the translations were appropriately reflecting the original phrasing. Participants were asked to make any corrections or changes to the items and responses on the translated versions of the questionnaires if they felt that they would improve the meaning and readability of the items. In addition, patients were asked to complete the questionnaire to assess distribution of responses. A study investigator then conducted interviews with participants using a series of standard questions, exploring the clarity of the questionnaire and individual items, and item acceptability and importance. Study materials were mailed or provided in person to participants, and follow-up interviews were conducted by telephone or face-to-face, depending on the participant preferences. Study data were entered into a Research Electronic Data Capture (REDCap) database. ${ }^{14}$

\section{Final Translation Process}

To generate the final versions of each questionnaire, the study coordinator collated feedback from all participants for each version and systematically reviewed the questionnaire with a hospital interpreter in the context of the feedback. Items that generated two or more suggested changes from participants were reviewed, and between the study coordinator and the interpreter, a consensus was agreed as to the changes to create the final questionnaire versions.

\section{Results}

\section{Participants}

\section{Traditional And Simplified Chinese}

Seven health professionals participated, six of whom assessed both the Traditional and Simplified Chinese versions, and one of whom assessed only the Traditional Chinese version of the instrument. Of these health professionals, three were nurses, two were colorectal surgeons, and two were oncologists (Table 1). Eleven Chinesespeaking patients participated; of whom, four assessed
Table I Characteristics Of Study Participants

\begin{tabular}{|c|c|c|}
\hline Health Professionals & $\begin{array}{l}\text { Arabic } \\
(n=6)\end{array}$ & $\begin{array}{l}\text { Chinese } \\
(n=7)\end{array}$ \\
\hline \multicolumn{3}{|l|}{ Sex } \\
\hline Male & 5 & 4 \\
\hline Female & I & 3 \\
\hline \multicolumn{3}{|l|}{ Profession } \\
\hline Surgeon & 3 & 2 \\
\hline Nurse & - & 3 \\
\hline Oncologist & I & 2 \\
\hline Gastroenterologist & I & - \\
\hline Health information manager & I & - \\
\hline Patients & $\begin{array}{l}\text { Arabic } \\
(n=8)\end{array}$ & $\begin{array}{l}\text { Chinese } \\
(n=I I)\end{array}$ \\
\hline \multicolumn{3}{|l|}{ Sex } \\
\hline Male & 6 & 6 \\
\hline Female & 2 & 5 \\
\hline \multicolumn{3}{|l|}{ Treatment } \\
\hline Surgery only & 3 & 4 \\
\hline Surgery + chemotherapy & 3 & 2 \\
\hline Surgery + radiotherapy & I & 1 \\
\hline Surgery + chemoradiotherapy & I & 3 \\
\hline Radiotherapy only & - & 1 \\
\hline
\end{tabular}

only the Traditional Chinese version, four only assessed the Simplified Chinese version, and three assessed both versions. Due to the consistencies in feedback between the Simplified and Traditional Chinese versions, we have presented feedback for these two written languages together in this manuscript to avoid repetition where possible.

\section{Arabic}

Six health professionals assessed the Arabic version of the CCCQ-P; of whom, three were surgeons, one was an oncologist, one gastroenterologist, and one health information manager. Eight Arabic-speaking patients assessed the Arabic version of the CCCQ-P.

\section{Instructions And Response Options}

Participants agreed that the instructions and response options presented in the CCCQ-P instrument were appropriate and clear, and no changes were suggested. There were some concerns among health professionals over the wording of questions to elicit demographic information, particularly around appropriate response options to categorise levels of education as these vary considerably between countries within language groups. However, as no patients reported difficulty with the response options, 
the only modification was to add "post-graduate degree" as a category. In general, participants who reviewed both the Simplified and Traditional Chinese versions regarded the Traditional Chinese version easier to read, as it explained questions and responses more clearly, particularly for medical terminology.

\section{Item Clarity, Comprehensiveness, And Significance}

Among both the Arabic and Traditional and Simplified Chinese versions, participants consistently identified medical terminology that was difficult to translate into Arabic or Chinese, and a consensus was not always reached. Examples of these terms included "multidisciplinary", "stoma" or "stomal therapist", "physiotherapist" or "palliative care". However, participants did agree that the meaning of the questions and response options was not lost, and majority of suggested changes were provided acknowledging that either option would be understood and would be a matter of preference. Minor changes to the wording of these questionnaires were made in response to this feedback.

\section{Item Importance And Acceptability}

Across all translated versions, no participants suggested the removal of any items based on inappropriateness, invasiveness, or redundancy, suggesting that participants agreed that the questions were important and should be retained. In assessing the acceptability, when asked to consider the length of the questionnaire, health professionals generally considered the questionnaire to be too lengthy; however, patients generally responded that the length was acceptable, with one participant drawing comparisons to other forms that are required to be completed during engagement with the health system.

\section{Discussion}

In this study, we undertook a rigorous process of translation of the CCCQ-P instrument into Chinese and Arabic, two of the commonest languages for culturally and linguistically diverse communities in Australia. The translations were found to have good face validity and provide a useful measure to assess migrant patients' experience of their cancer care in clinical and research settings. Additionally, the testing of additional items alongside the 20-item CCCQ$\mathrm{P}$ that were specific to migrant patients was considered valuable by participants, as they were specific to the experience of migrants using the Australian health care system. Though not part of the CCCQ-P itself, these items will be included alongside the CCCQ-P in future iterations in both English and non-English versions. This is the first step in understanding the migrant perspective of cancer care coordination in the Australian health system.

Both patients and health professionals perceived that a tool to measure experience of cancer care in a migrant patients' native language was appropriate and useful, although it was noted that some patients may be unaccustomed to rating their care in such a way in a new health system. Across both languages, there was some difficulty in achieving consensus on complex medical terminology, such as "stoma", "palliative care", and "multidisciplinary" during the initial forward and back translations, and during face validation with patients and health professionals. However, participants agreed that despite individual preferences for terms for medical terminology, the meaning of the words and phrases was not lost. Similar to Zeneli et al, ${ }^{15}$ who translated the Supportive Care Needs Survey Short Form 34 (SCNS-SF34) from English into Italian, as well as published optimal methodology for crosscultural adaptation of survey instruments, ${ }^{16,17}$ we utilised forward and back translations and expert committee reviews before conducting face validation with both patients (the end-users) and health professionals native in each respective language. As Gjersing et $\mathrm{al}^{16}$ recommend, appropriate translation and cross-cultural adaptation of any instrument is essential before being used in new settings if they intend to measure the same items as the original instrument. The acceptability of the translated versions of the CCCQ-P in Chinese and Arabic is promising for conduct of a large-scale psychometric validation study of the instruments in the Australian health care system. Future research may also conduct validation of the tools in countries with native speakers of Chinese and Arabic to produce a tool that can be adapted for the local health care system, which has strong psychometric properties ${ }^{17}$ in line with the original English CCCQ-P.

A primary strength of this study is the diversity of participants who provided feedback on the questionnaire. By approaching both patients and health professionals with varying degrees of experience with the health care system, length of residence in Australia, and demographic and clinical factors, we were able to produce a final facevalidated tool that was comprehendible, usable, and relevant to the population of interest. Patients reported a range of treatments, including unimodal and multimodal therapy (Table 1), and health professionals came from a representative group of health workers. Further, the inclusion of 
health professionals who provided feedback on the translated documents was considered critical, as these bilingual clinicians often converse with their migrant patients in their native language. Therefore, their perspective on how they may phrase difficult medical terminology during discussions with patients provided valuable insight into item clarify and comprehensiveness. A limitation of this study is that patients were recruited from one hospital in Sydney, affiliated with a comprehensive cancer centre, and so may not reflect the experience of all patients who are treated for cancer in New South Wales or Australia. Formal psychometric evaluation of the translated questionnaires was beyond the scope of the present study but could be undertaken using a larger sample of Chinese- and Arabic-speaking patients in the future.

Well-coordinated cancer care is a cornerstone of health care in Australia, with the "Optimal Care Pathways"18 indicating this should be an outcome to be focused on along with traditional outcomes such as survival. As such, policy makers may consider adopting the CCCQ-P into general usage in Australian cancer treatment centres. Translations of the CCCQ-P could enable further research to better understand the experience of migrant patients and to develop and test tailored strategies to improve care coordination for these groups. Furthermore, the availability of patient-reported measures in different languages allows participation of migrant patients in clinical trials and other research studies, thereby improving the generalisability of subsequent findings.

\section{Conclusions}

The Arabic and Chinese translations of the CCCQ-P instrument were well-received among both patients and health professionals and have potential to aid our understanding of the migrant patient experience of the coordination of their cancer care.

\section{Ethics Approval And Consent To Participate}

Ethical approval for this study was granted by the Sydney Local Health District Human Research Ethics Committee (SLHD HREC) (Protocol No X17-0230 \& HREC/17/ $\mathrm{RPAH} / 345$ ) and clinical governance was approved by the Research Governance Office at the Royal Prince Alfred Hospital. Written informed consent was obtained from all participants in this study.

\section{Abbreviations}

CALD, culturally and linguistically diverse; CCCQ-P, Cancer Care Coordination Questionnaire for Patients; HREC, Human Research Ethics Committee; NSW, New South Wales; PROM, patient-reported outcome measure; REDCap, Research Electronic Data Capture; SCNS-SF34, Supportive Care Needs Survey Short Form 34; SLHD, Sydney Local Health District.

\section{Acknowledgments}

This study was funded through Cancer Institute NSW Translational Health Services Research Program Grant No 09/THS/2-12 (awarded to Professor Jane M Young). The authors wish thanks the Sydney Health Care Interpreter Service for their assistance in developing final versions of the study questionnaires. The abstract was presented at the 1st World Congress on Migration, Ethnicity, Race and Health, 17th-19th May 2018, Edinburgh, Scotland. The Poster was published as Young J, Venchiarutti R, Durcinoska I, Steffens D. (2018) Measuring the experience of cancer care coordination among Chinese- and Arabic-speaking people in Sydney, Australia: adaptation and pilot testing of the CCCQ-P questionnaire. European Journal of Public Health 28 (S1):3.11-P6 DOI: doi.10.1093/eurpub/cky048.128.

\section{Author Contributions}

JMY designed the study. RLV collected data and wrote the first draft of the manuscript. JMY, DS, and ID revised the first draft into the final manuscript. All authors read and approved the final manuscript. All authors contributed to data analysis, drafting and revising the article, gave final approval of the version to be published, and agree to be accountable for all aspects of the work.

\section{Disclosure}

Jane Young reports grants from Cancer Institute NSW, during the conduct of the study. The authors report no other conflicts of interest in this work.

\section{References}

\footnotetext{
1. Durcinoska I, Young JM, Solomon MJ. Patterns and predictors of colorectal cancer care coordination: a population-based survey of Australian patients. Cancer. 2017;123(2):319-326. doi:10.1002/ cncr.30326

2. IOM. Delivering High-Quality Cancer Care: Charting a New Course for a System in Crisis. Washington (DC): The National Academic Press; 2013.
} 
3. McDonald KM, Sundaram V, Bravata DM, et al. Care coordination. In: Shojania KG et al. editors. Closing the Quality Gap: A Critical Analysis of Quality Improvement Strategies. Technical Review 9 (Prepared by the Stanford University-UCSF Evidence-based Practice Center under contract 290-02-0017). Rockville (MD): Agency for Healthcare Research and Quality; June 2007.

4. Snyder CF, Aaronson NK, Choucair AK, et al. Implementing patientreported outcomes assessment in clinical practice: a review of the options and considerations. Qual Life Res. 2012;21(8):1305-1314. doi:10.1007/s11136-011-0054-x

5. Australian Bureau of Statistics. 2071.0 - census of population and housing: reflecting Australia - stories from the census, 20162018. Available from: http://www.abs.gov.au/ausstats/abs@.nsf/Lookup/by $\%$ 20Subject/2071.0 2016 Main\%20Features Cultural\%20Diversity \%20Article 60. Accessed October 10, 2019.

6. Australian Bureau of Statistics. 2071.0 - reflecting a nation: stories from the 2011 census, 2012-2013. 2017. Available from: http://www. abs.gov.au/ausstats/abs@.nsf/Lookup/2071.0main+features9020122013. Accessed October 10, 2019.

7. Shaw JM, Shepherd HL, Durcinoska I, et al. It's all good on the surface: care coordination experiences of migrant cancer patients in Australia. Support Care Cancer. 2016;24(6):2403-2410. doi:10.1007/ s00520-015-3043-8

8. Butow PN, Bell ML, Aldridge LJ, et al. Unmet needs in immigrant cancer survivors: a cross-sectional population-based study. Support Care Cancer. 2013;21(9):2509-2520. doi:10.1007/s00520-013-1819-2

9. Sze M, Butow P, Bell M, et al. Migrant health in cancer: outcome disparities and the determinant role of migrant-specific variables. Oncologist. 2015;20(5):523-531. doi:10.1634/theoncologist.2014-0274

10. Ayanian JZ, Zaslavsky AM, Guadagnoli E, et al. Patients' perceptions of quality of care for colorectal cancer by race, ethnicity, and language. J Clin Oncol. 2005;23(27):6576-6586. doi:10.1200/jco.2005.06.102
11. Ayanian JZ, Zaslavsky AM, Arora NK, et al. Patients' experiences with care for lung cancer and colorectal cancer: findings from the Cancer Care Outcomes Research and Surveillance Consortium. J Clin Oncol. 2010;28(27):4154-4161. doi:10.1200/ jco.2009.27.3268

12. Young JM, Walsh J, Butow PN, et al. Measuring cancer care coordination: development and validation of a questionnaire for patients. BMC Cancer. 2011;11:298. doi:10.1186/1471-2407-11-298

13. Beaton DE, Bombardier C, Guillemin F, et al. Guidelines for the process of cross-cultural adaptation of self-report measures. Spine (Phila Pa 1976). 2000;25(24):3186-3191. doi:10.1097/00007632200012150-00014

14. Harris PA, Taylor R, Thielke R, et al. Research electronic data capture (REDCap)-a metadata-driven methodology and workflow process for providing translational research informatics support. $J$ Biomed Inform. 2009;42(2):377-381. doi:10.1016/j.jbi.2008.08.010

15. Zeneli A, Fabbri E, Donati E, et al. Translation of Supportive Care Needs Survey Short Form 34 (SCNS-SF34) into Italian and cultural validation study. Support Care Cancer. 2016;24(2):843-848. doi:10.1007/s00520-015-2852-0

16. Gjersing L, Caplehorn JR, Clausen T. Cross-cultural adaptation of research instruments: language, setting, time and statistical considerations. BMC Med Res Methodol. 2010;10:13. doi:10.1186/14712288-10-13

17. Terwee CB, Bot SD, de Boer MR, et al. Quality criteria were proposed for measurement properties of health status questionnaires. J Clin Epidemiol. 2007;60(1):34-42. doi:10.1016/j.jclinepi.2006.03.012

18. Cancer Council Victoria. Optimal Care Pathways. 2018. [Cited January 15 2019]. Available from: https://www.cancervic.org.au/forhealth-professionals/optimal-care-pathways. Accessed October 10, 2019
Patient Preference and Adherence

\section{Publish your work in this journal}

Patient Preference and Adherence is an international, peer-reviewed, open access journal that focusing on the growing importance of patient preference and adherence throughout the therapeutic continuum. Patient satisfaction, acceptability, quality of life, compliance, persistence and their role in developing new therapeutic modalities and compounds to optimize clinical outcomes for existing disease states are major areas of interest for the journal. This journal has been accepted for indexing on PubMed Central. The manuscript management system is completely online and includes a very quick and fair peer-review system, which is all easy to use. Visit http:// www.dovepress.com/testimonials.php to read real quotes from published authors. 